\title{
LabVIEW Based Implementation of Resistive Temperature Detector Linearization Techniques
}

\author{
Sidi M. Ahmed Ghaly \\ Electrical Engineering Department \\ College of Engineering \\ Al Imam Mohammad Ibn Saud Islamic University (IMSIU) \\ Riyadh, Saudi Arabia \\ smghaly@imamu.edu.sa
}

\begin{abstract}
Resistance temperature detectors (RTDs) are highquality temperature sensors used for accurate temperature measurements and ideally suited for industrial applications, but their non-linearity is a serious drawback in temperature monitoring in which precise measurement and control are crucial. In this paper, two linearization techniques are implemented in LabVIEW environment involving voltage divider and feedback compensation circuits. The presented techniques considerably decrease the effects of non-linearity and may accommodate temperature variations).
\end{abstract}

Keywords-RTD; LabVIEW; Wheatstone bridge; instrumentation amplifier; simulation; sensor

\section{INTRODUCTION}

Resistance temperature detectors (RTDs), as their name implies, are sensors that measure the temperature by correlating the resistance of the RTD element with it. Most RTD elements consist of a certain length of very thin wire surrounded by a ceramic or glass core $[1,2]$. The element is usually quite fragile, and it is often placed inside a sheathed probe for protection. The RTD element is made from a pure material, whose resistance to different temperatures has been attested [3, 4]. RTD is one of the most accurate temperature sensors. It offers a good level of accuracy and is extremely stable and reliable. RTDs are also relatively insensitive to electrical noise and therefore well suited for temperature measurements in industrial environments, especially around motors, generators and other high voltage equipment. The resistance of an RTD sensor increases with the temperature by about $0.39 \% /{ }^{\circ} \mathrm{C}$, but they have a significant non-linearity of about $0.38 \%$ for an interval of $100^{\circ} \mathrm{C}$ [5]. This non-linearity is often numerically corrected, but there are many applications to correct it analogically. RTDs can be used for many industrial applications because they are inexpensive. However, their nonlinear resistance characteristic makes accurate temperature conversion complex. To minimize this difficulty, the RTD's resistance characteristic should be linearized by placing a thermistor within a resistor divider or with feedback compensation. This linearized response is simpler to convert to a temperature value than the thermistor's raw response. To obtain an optimum level of linearity, a pair of formulas can be

Corresponding author: Sidi M. Ahmed Ghaly derived as will be explained below. These formulas are useful in determining component values for this common linearization circuit. When the RTD is excited with a voltage source, the resulting RTD voltage is directly proportional to the resistance, producing the same nonlinearity. However, with the use of a resistor divider, Wheatstone bridge, or feedback compensation, the non-linearity can be significantly reduced, and this linearization technique can be applied to other resistive sensors, mainly with second-order non-linearity. The law of variation of the resistance with the temperature for the RTD sensors is given by the formula of approximate relation [6]:

$$
R_{T D}(T)=R_{0}\left(1+A T+B T^{2}+C(\mathrm{~T}-100) T^{3}\right)
$$

where $A=3.908 \times 10^{-3}, B=-5.802 \times 10^{-7}$ and $C=0$ for $T<0$.

To obtain an RTD resistance that varies almost linearly with temperature, two linearization methods are presented in this paper using the Laboratory Virtual Instrument Engineering Workbench (LabVIEW) environment which allows developing a program for the design and analysis of the RTD linearization circuits [7-9]. Firstly, a voltage divider can be used by placing a resistor $R$ in series with the RTD. Then, a Wheatstone bridge with feedback compensation is implemented. The bridge output varies in a non-linear manner with a variation of temperature of the process parameter, and this non-linearity factor can be eliminated by incorporating feedback in the bridge amplifier or by software means [10]. But for both methods, the combination of resistance tolerance and temperature coefficient defines the resistance against the temperature characteristics for the RTD sensor. The greater the tolerance of the element, the more the sensor will spread a generalized curve, and the more variation it will be to the sensor. This is important for users who need to change or replace sensors and want to minimize systematic errors. This work may offer a beneficial base for engineers who are trying to reduce the cost and time of RTD-related circuits and can also be applied for high precision temperature sensing in MRI system applications [11-14].

\section{LINEARIZATION TECHNIQUES}

\section{A. Linearization with Voltage Divider Method}

To obtain an output voltage $V_{o}$ that varies almost linearly with temperature, a voltage divider can be made by placing a 
resistor $R$ in series with the RTD [15]. However, the major problem is how to determine the optimal resistance value that will attain the expected linear range. The ratio between the output and the input voltage can be expressed as:

$$
\frac{V_{o}}{V_{i}}=g(T)=\frac{1}{1+m \times(T)}
$$

where $m$ is the ratio of $R_{o}$ to $R$, and $x(T)$ is the ratio of $R_{T D}$ to $R_{o}$

The function $g(T)$ is represented in Figure 1 in terms of temperature for different values of $m$. The expected temperature range is from $0^{\circ} \mathrm{C}$ to $180^{\circ} \mathrm{C}$.

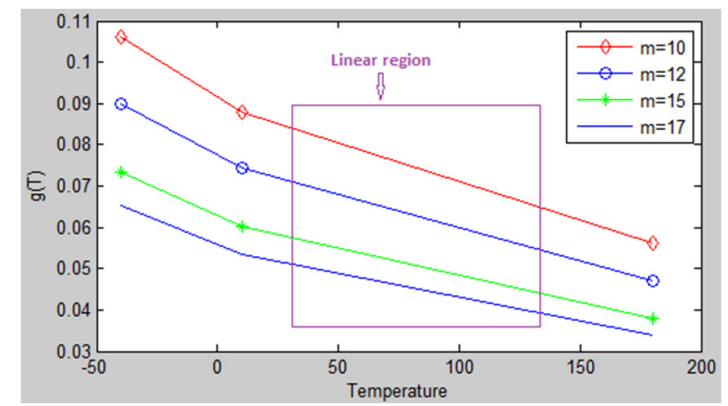

Fig. 1. Curves of $g(T)$ for different values of $m$

It is clear from Figure 1 that as the factor $m$ increases, the linear region decreases and gets limited. The appropriate value of $m$ can be determined to increase the linear region using the second derivative of the function $g(T)$ that can be solved as:

$$
\frac{d^{2} g(T)}{d T^{2}}=\frac{-\frac{d x(T)}{d T}}{(1+\mathrm{m} \times(T))^{2}}
$$

to get a straight-line response for $g(T)$. The appropriate value of $m$ can be found from the following expression:

$$
m=\frac{2 B}{\left(1+\mathrm{AT}+\mathrm{B} T^{2}\right)\left[(\mathrm{A}+2 \mathrm{BT})^{2}-2 B\right]}
$$

\section{B. RTD Linearization with Feedback Compensation}

One of the methods to avoid the non-linearity problem is the use of bridge measurement. Wheatstone bridge measurement is an indirect indication of the resistance of the RTD. The bridge requires four connecting wires, an external source, an amplifier, and three resistors that have a temperature coefficient of zero. To avoid subjecting the other three resistors of the bridge to the same temperature, the RTD is separated from the bridge by a pair of connecting wires. These wires recreate the problem of non-linearity: The impedance of the connecting wires affects the reading of the temperature. This effect can be minimized by choosing a large value for the ratio of $R 1$ to $R 3$. However, the sensitivity of the bridge should be decreased, resulting in low bridge output and hence, low resolution. The bridge output voltage $V_{o}$ can be expressed as:

$$
V o=A \operatorname{Vin}\left(\left(\frac{R_{T D}}{R_{T D}+R 2}\right)-\left(\frac{R 3}{R 3+R 1}\right)\right)
$$

where $R_{T D}$ is the resistance of the RTD at temperature $T, V_{i n}$ is the input voltage, and $A$ is the gain of the amplifier.
Rearranging (5), the output voltage is expressed in terms of the ratio $R 1$ to $R 3$ as:

$$
V o=A \operatorname{Vin}\left(\left(\frac{R_{T D}}{R_{T D}+R 2}\right)-\left(\frac{1}{1+\left(\frac{R 1}{R 3}\right)}\right)\right)
$$

Thus, the simple Wheatstone bridge creates a non-linear relationship between the resistance change and the bridge voltage change, which requires a feedback compensation scheme that can be used for the linearization of the bridge output. The proposed diagram is shown in Figure 2. The bridge output $V_{o b}$ is amplified by an instrumentation amplifier of gain $A$ to generate the final output, $V o$. A part of the output is added to a reference voltage $V_{r}$ using a unity gain adder, and the output of the adder delivers the source excitation, $V_{\text {in }}$ [16].

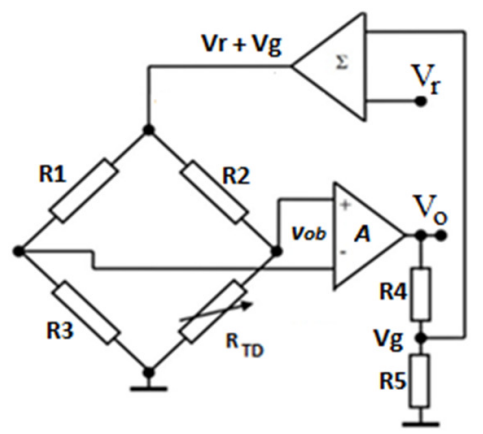

Fig. 2. Feedback compensation configuration

It is clear from the feedback compensation circuit that the output voltage $V_{0}$ is expressed as:

$$
V o=A V o b=A(V r+V g)\left(\left(\frac{R_{T D}}{R_{T D}+R 2}\right)-\left(\frac{1}{1+\left(\frac{R 1}{R 3}\right)}\right)\right)
$$

where $V g=(R 5 /(R 5+R 4)) V o$, and $V r$ is the reference voltage. Rearranging (7), the output voltage $V_{0}$ is expressed as:

$$
V o=A\left(V r+\frac{R 5}{R 5+R 6} V o\right)\left(\left(\frac{R_{T D}}{R_{T D}+R 2}\right)-\left(\frac{1}{1+\left(\frac{R 1}{R 3}\right)}\right)\right)
$$

If the factor of attenuation $\frac{R 5}{R 5+R 6}$ is chosen such that $\frac{A R 5}{R 5+R 6}$ equals to $\frac{R 1}{R 3}+1$ then (8) implies that the feedback scheme of Figure 4 provides an amplified output, linearly proportional to the temperature expressed as:

$$
V o=A V r \frac{\frac{R 1}{R 3}}{\left(1+\left(\frac{R 1}{R 3}\right)\right)^{2}}(1+\alpha T)
$$

Figrue 3 shows the output voltage as a function of temperature for different values of the $R I / R 3$ ratio.

A series of simulations were carried out in Labview to determine the efficacy of the proposed method and are detailed below. 


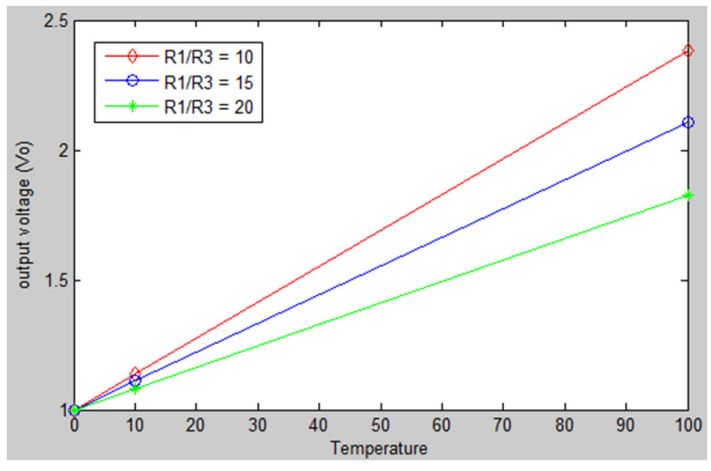

Fig. 3. Output Voltage curves for different values of $R 1 / R 3$

\section{SOFTWARE DEVELOPMENT AND SIMULATION RESULTS}

The virtual instrument concept that gave LabVIEW its name is manifested by the permanence of a graphical interface for each module (function) of a program. The controls and indicators on the front panel are the interfaces through which the program interacts with the user (reading commands and parameters, displaying results). The control-command functions of cards or instruments are the interface through which the program interacts with the editing [7]. Both presented linearization techniques were implemented and incorporated into a virtual instrument on the LabVIEW environment. Figure 4 shows the block diagram of the implementation process.

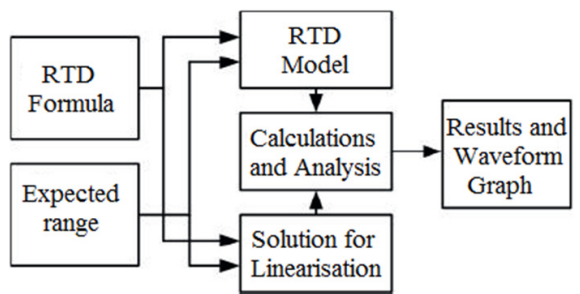

Fig. 4. Block diagram of the implementation process

The implementation includes the specified and defined RTD character and the expected temperature range. Also, by the other described conditions, an optimal linear region or solution for linearization can be obtained. Finally, the "waveform graph" output on the front panel can display simulation results and temperature plots in the expected range. Figures 5 and 6 show the block diagram and the front panel (temperature waveform graph) that follows the divider voltage model presented in Figure 1. In this block diagram, three sub virtual instruments (SubVis) are created to specify the RTD character and perform the required calculations (optimal value of $R$ and excepted linear region). The program gives the optimal value of $R$ is 50k for linearizing a RTD over the range of $0-180^{\circ} \mathrm{C}$. Figures 7 and 8 show the block diagram and the interactive user interface of the VI (front panel of the system) that follows the feedback compensation model presented in Figure 3.

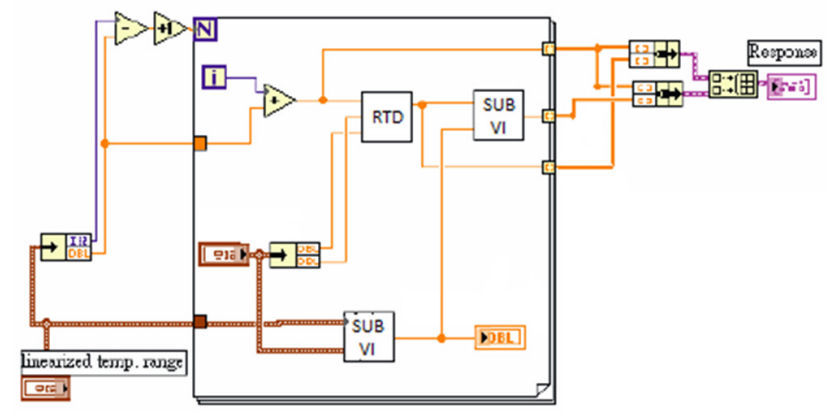

Fig. 5. Block diagram: Voltage divider method

It is clear from Figure 8 that we can get a straight-line curve of RTD. This linearity is good relatively to the one obtained with the divider voltage method. Simulation results show that the non-linearity decreases when the value of amplifier gain increases up to a certain limit. After that limit, non-linearity increases and the change will be on the value of the output voltage since the amplifier gain is multiplied by it and then the relation between temperature and Voltage will be changed as shown in Figure 9.

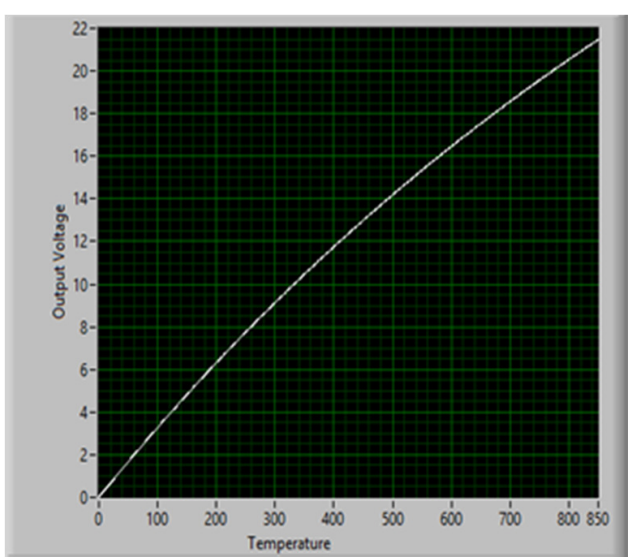

Fig. 6. Waveform graph of the output Voltage in terms of temperature for divider voltage circuit

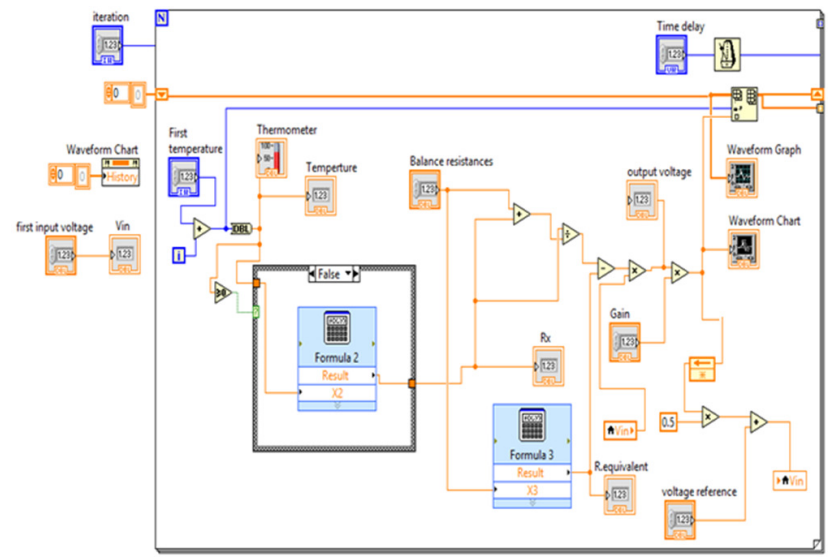

Fig. 7. Block diagram: Feedback compensation method 


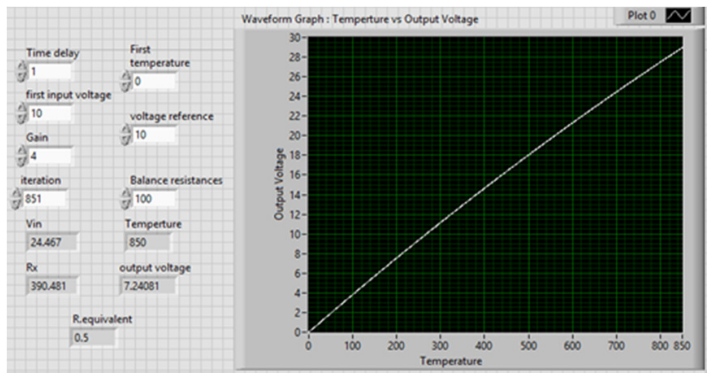

Fig. 8. Interactive user interface for feedback compensation method

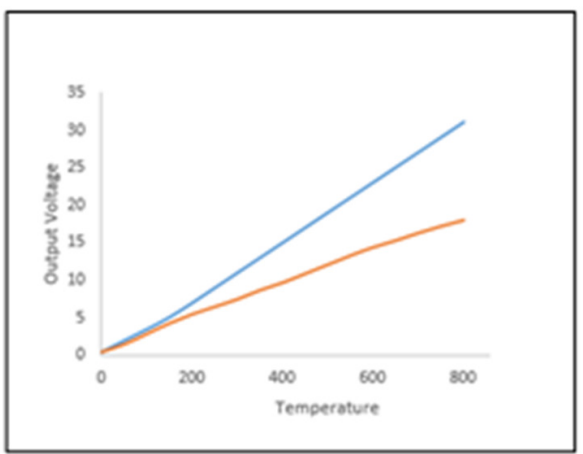

Fig. 9. Output Voltage curves for two values of amplifier gain $A$ with feedback compensation.

\section{CONCLUSION}

RTD linearization has been implemented in LabVIEW, using divider voltage and feedback compensation methods. A two-parameter model for RTD was studied and presented to simulate and implement linearization techniques within an expected temperature range from $0^{\circ} \mathrm{C}$ to $500^{\circ} \mathrm{C}$. The linearization error was estimated to be less than $0.5^{\circ} \mathrm{C}$. By comparison with a resistance RTD without linearization, this represents an improvement of around $25 \%$, which is very important for industrial applications like thermal compensation and room temperature measurement. As a final conclusion, LabVIEW is an effective tool for analyzing RTD to define an optimal value for a resistor used in RTD linearization.

\section{REFERENCES}

[1] B. E. Noltingk, Instrumentation Reference Book, ButterworthHeinemann, 1988

[2] S. K. Sen, "An improved lead wire compensation technique for conventional two-wire resistance temperature detectors (RTDs)", Measurement, Vol. 39, No. 5, pp. 477-480, 2006

[3] F. N. Trofimenkoff, A. E. Nordquist, "Single amplifier resistance bridges with feedback linearization", IEEE Transactions on Instrumentation and Measurement, Vol. 33, No. 1, pp. 60-63, 1984

[4] Omega Engineering, Omega Complete Temperature Measurement Handbook and Encyclopedia, Omega Engineering, 1988

[5] B. Trump, "Analog linearization of resistance temperature detectors", Analog Application Journal, Vol. 4Q, pp. 21-24, 2011

[6] S. Pradhan, S. Sen, "An improved lead compensation technique for three-wire resistance temperature detectors", IEEE Transactions on Instrumentation and Measurement, Vol. 48, No. 5, pp. 903-905, 1999

[7] C. J. Kalkman, "LabVIEW: A software system for data acquisition, data analysis, and instrument control", Journal of Clinical Monitoring, Vol. 11, No. 1, pp. 51-58, 1995
[8] A. Gani, M. J. E. Salami, "A LabVIEW Based Data Acquisition System for Vibration Monitoring and Analysis", Student Conference on Research and Development, Shah Alam, Malaysia, July 17, 2002

[9] F. Alorifi, S. M. A. Ghaly, M. Y. Shalaby, M. A. Ali, M. O. Khan, "Analysis and detection of a target gas system based on TDLAS \& LabVIEW", Engineering, Technology \& Applied Science Research, Vol. 9, No. 3, pp. 4196-4199, 2019

[10] S. M. A. Ghaly, Antennes Radiofrequence Pour IIRM, Theorie et Application: Etude de Systemes Multispire Derives des Bobines de Helmholtz-Application a IIRM, Editions Universitaires Europeennes, 2015

[11] S. M. A. Ghaly, S. A. Sowayan, "A high B1 field homogeneity generation using free element elliptical four-coil system", American Journal of Applied Sciences, Vol. 11, No. 4, pp. 534-540, 2014

[12] S. M. A. Ghaly, K. A. A. Snaie, S. A. Sowayan, "Design and testing of radiofrequency spherical”, Modern Applied Science, Vol. 10, No. 5, pp. 186-193, 2016

[13] S. M. A. Ghaly, K. A. A. Snaie, O. K. Mohammad, "Spherical and improved helmholtz coil with high B1 homogeneity for magnetic resonance imaging", American Journal of Applied Sciences, Vol. 13, No. 12, pp. 1413-1418, 2016

[14] S. M. A. Ghaly, K. A. A. Snaie, A. M. Ali, "Design and modeling of a radiofrequency coil derived from a helmholtz structure", Engineering, Technology \& Applied Science Research, Vol. 9, No. 2, pp. 4037-4043, 2019

[15] S. K. Sen, T. K. Pan, P. Ghosal, “An improved lead wire compensation technique for conventional four-wire resistance temperature detectors (RTDs)", Measurement, Vol. 44, No. 5, pp. 842-846, 2011

[16] P. R. Nagarajan, B. George, V. J. Kumar, "A linearizing digitizer for wheatstone bridge based signal conditioning of resistive sensors", IEEE Sensors Journal, Vol. 17, No. 6, pp. 1696-1705, 2017 